Article

\title{
Negative Correlation between Lipid Content and Antibiotic Activity in Streptomyces: General Rule and Exceptions
}

\author{
Michelle David ${ }^{1, \dagger}$, Clara Lejeune ${ }^{1,+}$, Sonia Abreu ${ }^{2}$, Annabelle Thibessard ${ }^{3}$, Pierre Leblond ${ }^{3}(\mathbb{D}$, \\ Pierre Chaminade ${ }^{2}$ and Marie-Joelle Virolle ${ }^{1, *}$ \\ 1 Institute for Integrative Biology of the Cell (I2BC), CEA, CNRS, Université Paris-Saclay, \\ 91198 Gif-sur-Yvette, France; michelle.david@i2bc.paris-saclay.fr (M.D.); \\ clara.lejeune@i2bc.paris-saclay.fr (C.L.) \\ 2 Lipides, Systèmes Analytiques et Biologiques, Université Paris-Saclay, 92296 Châtenay-Malabry, France; \\ sonia.abreu@universite-paris-saclay.fr (S.A.); pierre.chaminade@universite-paris-saclay.fr (P.C.) \\ 3 Université de Lorraine, INRAE, DynAMic, F-54000 Nancy, France; \\ annabelle.thibessard@univ-lorraine.fr (A.T.); pierre.leblond@univ-lorraine.fr (P.L.) \\ * Correspondence: marie-joelle.virolle@i2bc.paris-saclay.fr \\ + These authors contributed equally to this work.
}

Received: 24 April 2020; Accepted: 20 May 2020; Published: 26 May 2020

\begin{abstract}
Streptomycetes are well known antibiotic producers and are among the rare prokaryotes able to store carbon as lipids. Previous comparative studies of the weak antibiotic producer Streptomyces lividans with its ppk mutant and with Streptomyces coelicolor, which both produce antibiotics, suggested the existence of a negative correlation between total lipid content and the ability to produce antibiotics. To determine whether such a negative correlation can be generalized to other Streptomyces species, fifty-four strains were picked randomly and grown on modified R2YE medium, limited in phosphate, with glucose or glycerol as the main carbon source. The total lipid content and antibiotic activity against Micrococcus luteus were assessed for each strain. This study revealed that the ability to accumulate lipids was not evenly distributed among strains and that glycerol was more lipogenic than glucose and had a negative impact on antibiotic biosynthesis. Furthermore, a statistically significant negative Pearson correlation between lipid content and antibiotic activity could be established for most strains, but a few strains escape this general law. These exceptions are likely due to limits and biases linked to the type of test used to determine antibiotic activity, which relies exclusively on Micrococcus luteus sensitivity. They are characterized either by high lipid content and high antibiotic activity or by low lipid content and undetectable antibiotic activity against Micrococcus luteus. Lastly, the comparative genomic analysis of two strains with contrasting lipid content, and both named Streptomyces antibioticus (DSM 41,481 and DSM 40,868, which we found to be phylogenetically related to Streptomyces lavenduligriseus), indicated that some genetic differences in various pathways related to the generation/consumption of acetylCoA could be responsible for such a difference.
\end{abstract}

Keywords: Streptomyces; antibiotics; lipids; triacylglycerol; metabolism; glycerol; glucose

\section{Introduction}

The Streptomyces genus is a well-known producer of most antibiotics in current use and of a variety of other bioactive molecules useful to human health (e.g., anti-cancer and anti-inflammatory drugs) or agriculture (e.g., fungicides, pesticides, insecticides and herbicides) [1-4]. Furthermore, Streptomyces is one of the rare prokaryotes to have the ability to accumulate storage lipids of the triacylglycerol family when cultivated under nitrogen and/or phosphate limitation [5]. The biosynthesis of these molecules 
requires the availability of glycerol3P and acetylCoA, both stemming from glycolysis. The condensation of acetylCoA results in fatty acid biosynthesis. The transfer of fatty acids on the glycerol backbone by glycerol3P acyl transferase (GPAT) yields diacylglycerol (DAG), a molecule precursor of polar membranous phospholipids as well as neutral lipids such as triacylglycerol (TAG). In previous studies, we demonstrated that, when grown on R2YE medium [6] limited in phosphate and containing glucose as the main carbon source, the weak antibiotic producer, S. lividans, had a high TAG content, indicative of a glycolytic metabolism [7]. By contrast, the $p p k$ mutant of $S$. lividans and the closely related model strain, S. coelicolor - which both produce the same antibiotics-have a low TAG content compared to the wild type strain of $S$. lividans $[7,8]$. These two strains suffer energetic stress. S. coelicolor suffers energetic stress since the two component system PhoR/PhoP that positively controls the phosphate supply, necessary for ATP synthesis, is weakly expressed in this strain [9] whereas the ppk mutant suffers energetic stress since its lacks PPK, an important enzyme that regenerates ATP from ADP and polyphosphate $[10,11]$. In these two strains, similar homeostatic processes are triggered to restore their energetic balance $[7,9]$. Such processes include the activation of their TCA cycle, which yields reduced co-factors whose re-oxidation by the respiratory chain generates ATP. In these strains, with acetylCoA being used to feed the TCA cycle, it is not available to be stored as lipids $[7,9]$. The activation of the oxidative metabolism of these strains was shown to be correlated with the production of the antibiotics CDA (calcium dependent antibiotic), RED (undecylprodigiosin) and ACT (actinorhoddin) [7]. CDA and RED were proposed to create damage to the membrane, contributing to the cell death and lysis of a fraction of the population $[7,12,13]$. This would provide nutrients, and especially phosphate, to support the activation of the oxidative metabolism of the surviving population. Furthermore, since the onset of ACT biosynthesis was shown to coincide with an abrupt drop in the intracellular ATP concentration in S. coelicolor, ACT, like other molecules possessing quinone groups (melanine, humic acid etc.), was proposed to act as an electron acceptor [7]. In a context of low phosphate availability, ACT would reduce the electron flow through the respiratory chain, reducing respiration efficiency and thus ATP generation, in order to adjust it to low phosphate availability [7,12].

A putative link between TAG content and antibiotic activity was already anticipated twenty years ago by Olukoshi and Packter $[14,15]$ in S. lividans. Furthermore, the impaired biosynthesis $[16,17]$ or enhanced degradation [18-20] of fatty acids was indeed shown to be correlated with increased antibiotic biosynthesis in S. coelicolor. Similarly, enhanced spiramycin biosynthesis was correlated with lower lipid content in S. ambofaciens [21]. In order to determine whether these observations constituted a general rule for Streptomyces species, fifty-four randomly picked Streptomyces strains from our collection were grown on modified solid R2YE medium limited in phosphate with either glucose or glycerol as the main carbon source. Their total lipid content, on glucose or glycerol, was assessed by Fourier transformed infra-red spectroscopy (FTIRS) [22-24], and the measure of the diameter of the growth inhibition zones around four agar plugs of each strain gave an estimation of their antibiotic activity against Micrococcus luteus (Figure 1). This study confirmed the existence of a negative correlation between lipid content and antibiotic activity but with some exceptions commented on in the discussion. It also revealed that glycerol was more lipogenic than glucose but was not as good a carbon source as glucose to promote antibiotic activity. An explanation concerning this unexpected observation is also proposed in the discussion. Lastly, a comparative analysis of the genome of two Streptomyces antibioticus strains-DSM 41,481 and DSM 40,868, bearing among the highest and lowest lipid contents on glycerol-was carried out in an attempt to determine the genetic features responsible for their highly different lipid contents. 


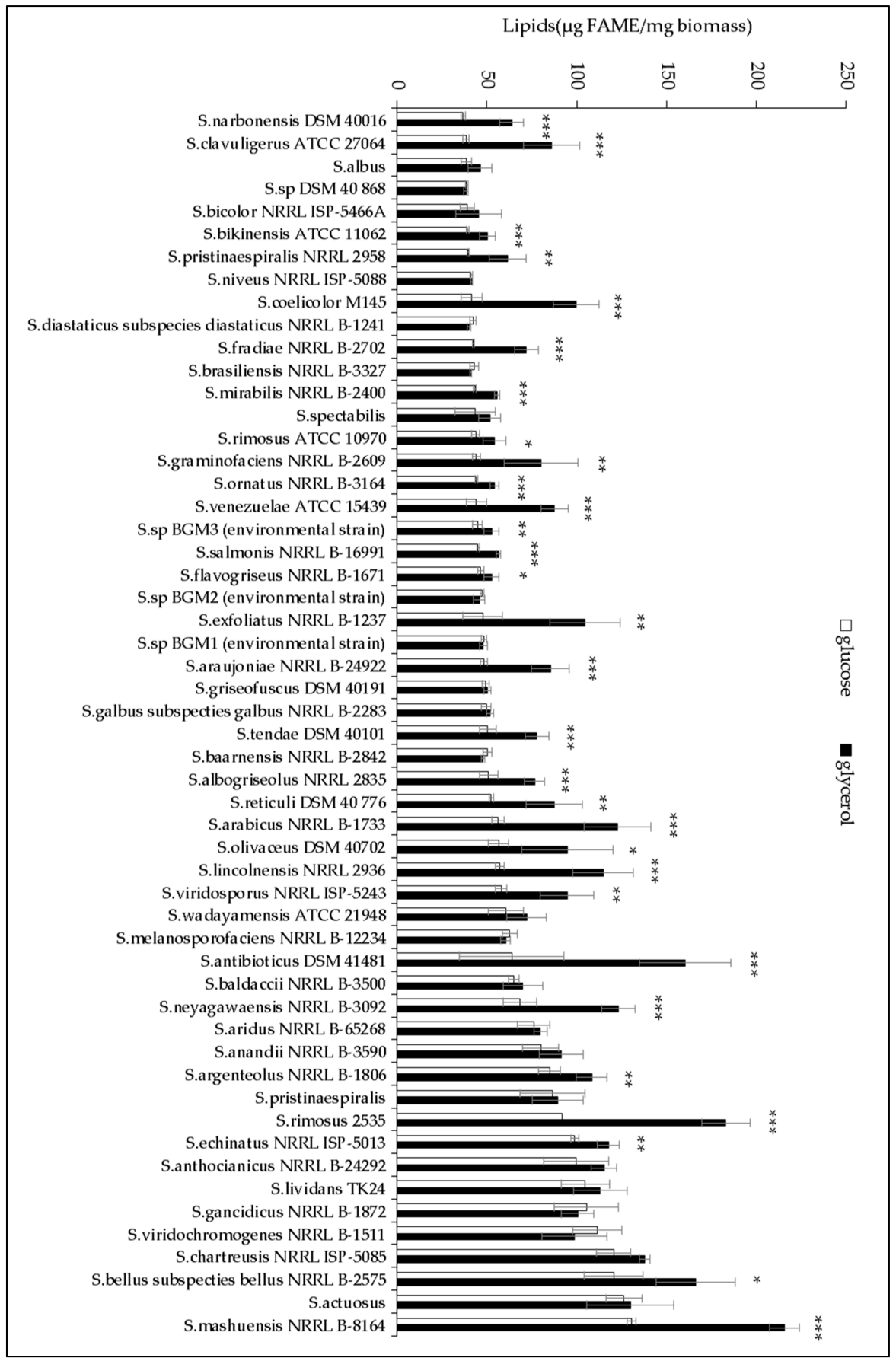

(A)

Figure 1. Cont. 


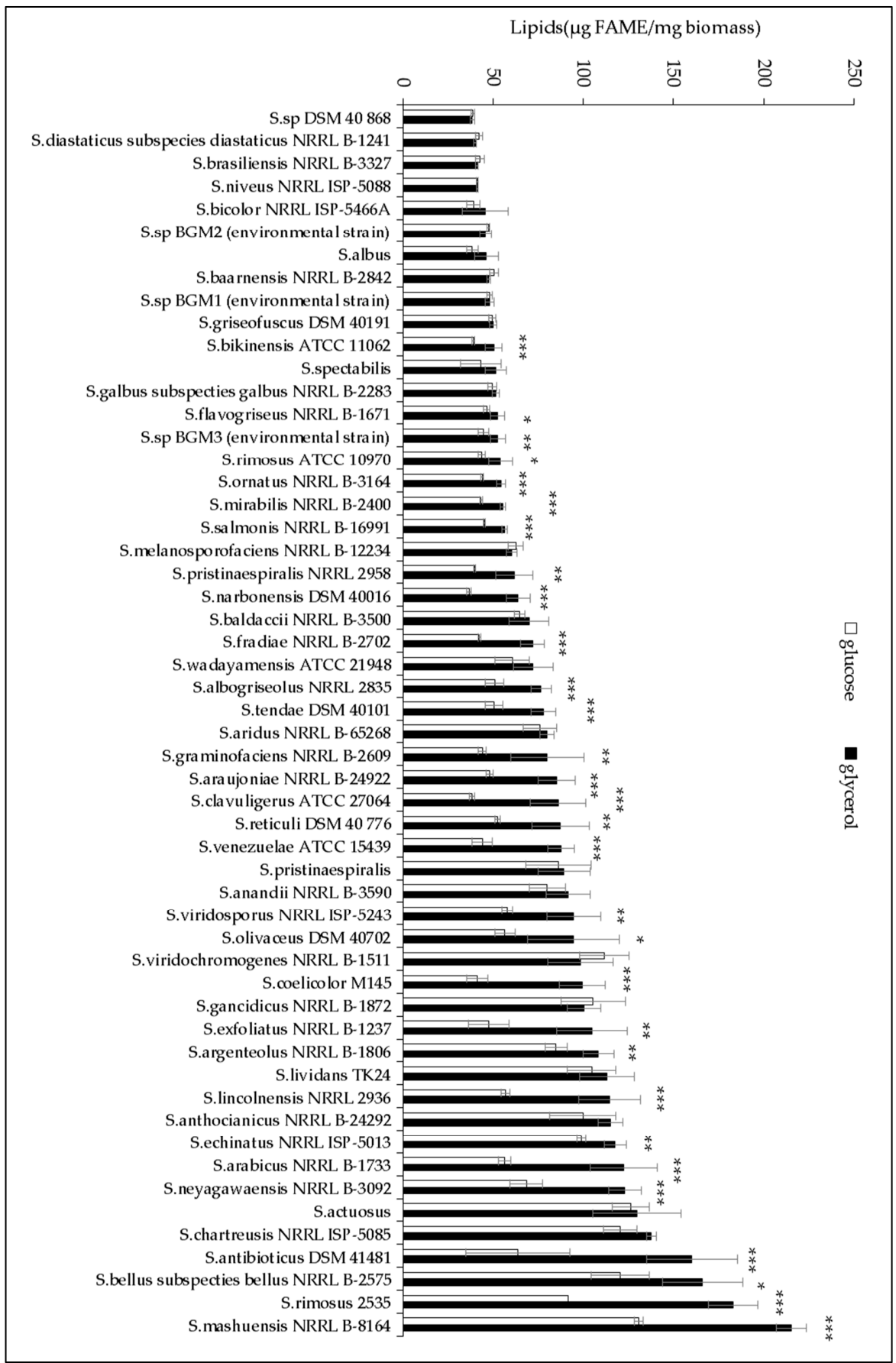

(B)

Figure 1. Cont. 


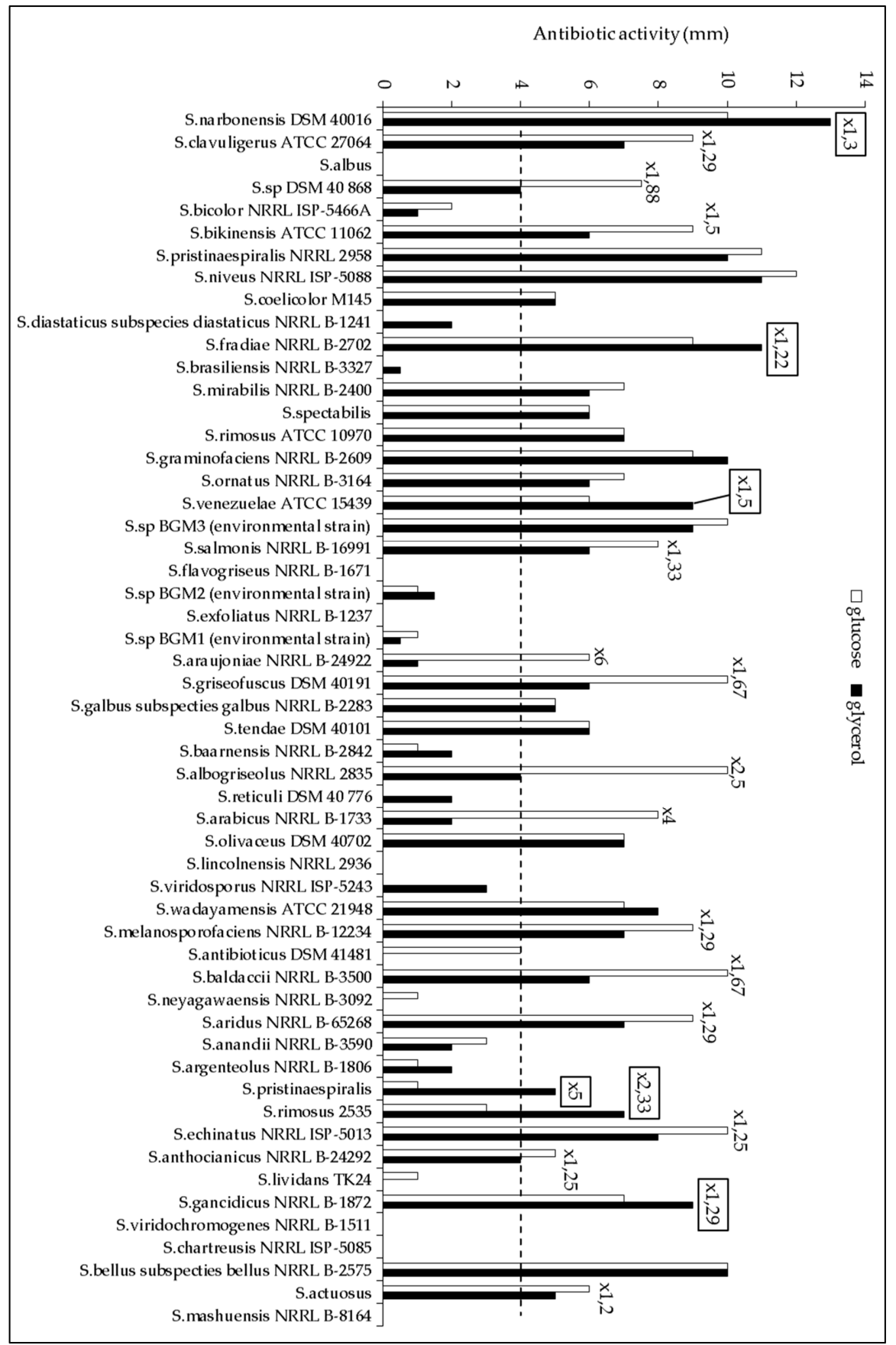

(C)

Figure 1. Total lipid/fatty methyl ester (FAME) content and antibiotic activity of Streptomyces strains. The strains were grown on modified solid R2YE limited in phosphate for $72 \mathrm{~h}$ at $28^{\circ} \mathrm{C}$ with either glucose (white histograms) or glycerol (black histograms) as the main carbon source. Their total lipid/FAME content, determined with FTIRS, was ranked according to their increasing lipid/FAME content upon growth on (A) glucose or on (B) glycerol. Significant differences in lipid content between glucoseand glycerol-grown cultures are represented by an asterisk (ANOVA, ${ }^{* * *}=p<0.001 ;{ }^{* *}=p<0.01$; $*=p<0.05$ ). (C) Antibiotic activity of Streptomyces strains determined by the measurement of the diameters of the zones of growth inhibition of Micrococcus luteus around four agar plugs of each Streptomyces culture. Fold changes in antibiotic activity ( $>1.2$ fold) between glucose and glycerol for inhibition zones $>4 \mathrm{~mm}$ are shown above the histograms. The fold changes of the six cases where antibiotic activity was higher on glycerol than on glucose are boxed. 


\section{Results}

2.1. The Lipid Content and Antibiotic Activity of the Streptomyces Strains Varies with the Nature of the Carbon Source Used for Growth

Fifty-four randomly picked Streptomyces strains from our collection were grown for $72 \mathrm{~h}$ at $28^{\circ} \mathrm{C}$ on the surface of cellophane disks deposited on the surface of the modified (no sucrose added) R2YE medium [6] limited in phosphate and containing either glucose (50 mM) or glycerol (100 mM) as the main carbon source. The lipid content of the strains was determined using the fast and reliable FTIRS method [22-24]. The FTIRS values, expressed as arbitrary units, were converted into $\mu \mathrm{g}$ of Fatty Methyl Esters (FAME) per mg of dry mycelium using the converting equation established by Millan-Oropeza et al. (2017) [24]. Indeed, in the presence of methanol, fatty acids constitutive of all cellular lipids are esterified to yield FAME that can be accurately quantified by GC/MS [22-24]. The presence of antibiotic activity excreted in the agar medium was assessed by the measurement of the diameters of the zones of growth inhibition of Micrococcus luteus around four agar plugs of each Streptomyces strain tested. The strains were ranked according to their lipid/FAME content on glucose (Figure 1a) or glycerol (Figure 1b), and their antibiotic activity is shown in (Figure 1c). This study revealed that the average lipid content of the strains was highly variable, revealing their highly different metabolic features, and was usually higher on glycerol than on glucose (Figures 1 and 2). It also revealed that some strains have far lower lipid content on glucose than on glycerol (Figure 1b). These strains might have, like $S$. coelicolor, an oxidative metabolism yielding an insufficient amount of glycerol3P to support high lipid biosynthesis on glucose [9]. Thirty and fifteen strains (forty-five strains) had a medium (50-100 $\mu \mathrm{g} / \mathrm{mg}$ ) or high (>100 $\mu \mathrm{g} / \mathrm{mg}$ ) lipid content on glycerol whereas only twenty and seven strains (twenty-seven strains) had medium or high lipid content on glucose, respectively. Consistently, the number of strains bearing a low lipid content $(<50 \mu \mathrm{g} / \mathrm{mg}$ ) was 3 fold higher on glucose (twenty-seven strains) than on glycerol (nine strains) (Figure 2). This confirmed that glycerol was more lipogenic than glucose, likely because the generation of glycerol3P, a major lipid precursor, from glycerol is more direct than that from glucose (one step versus five steps).

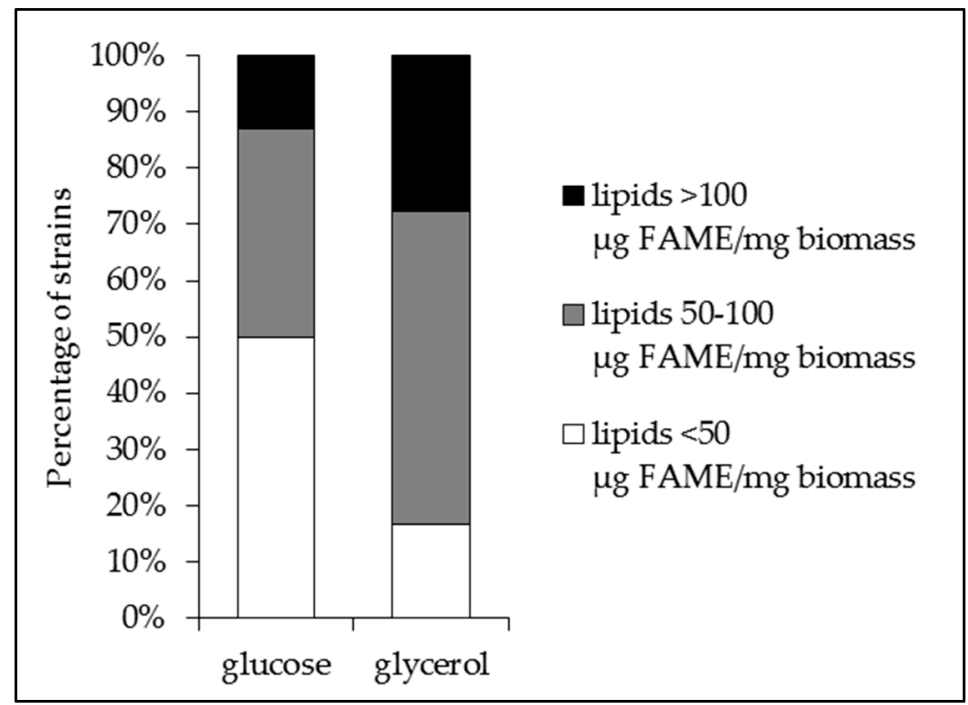

Figure 2. Percentage of Streptomyces strains grown for $72 \mathrm{~h}$ at $28{ }^{\circ} \mathrm{C}$ on solid modified R2YE limited in phosphate with either glucose $(50 \mathrm{mM})$ or glycerol $(100 \mathrm{mM})$ as the main carbon source bearing low ( $<50 \mu \mathrm{g}$ FAME/mg biomass, white part); medium (50-100 $\mu \mathrm{g}$ FAME/mg biomass, grey part) and high ( $>100 \mu \mathrm{g}$ FAME/mg biomass, black part) total lipid/FAME content.

This study also revealed that the antibiotic activity of the strains was also highly variable and was usually higher on glucose than on glycerol (Figure 3a,c). On glucose, fifteen strains gave halos of 
inhibition $>8 \mathrm{~mm}$, and among them, nine $(60 \%)$ had a low lipid content, five $(33.3 \%)$ had a medium lipid content and one (6.7\%) had a high lipid content. By contrast, on glycerol, only nine strains gave halos of inhibition $>8 \mathrm{~mm}$, and among them, only one $(11.1 \%)$ had a low lipid content, six (66.7\%) had a medium lipid content and two $(22.2 \%)$ had a high lipid content (Figure 3a,c). Furthermore, among the thirty-four strains that gave inhibition halos with diameters greater than or equal to $4 \mathrm{~mm}$, fourteen $(41.2 \%)$ gave halos of similar size on both carbon sources, fourteen $(41.2 \%)$ gave bigger halos on glucose than on glycerol and only six (17.6\%) gave bigger halos on glycerol than on glucose (Figure 1c). This suggested that glycerol does not promote as good antibiotic activity as glucose. A hypothesis concerning the cause of this difference is proposed in the discussion.

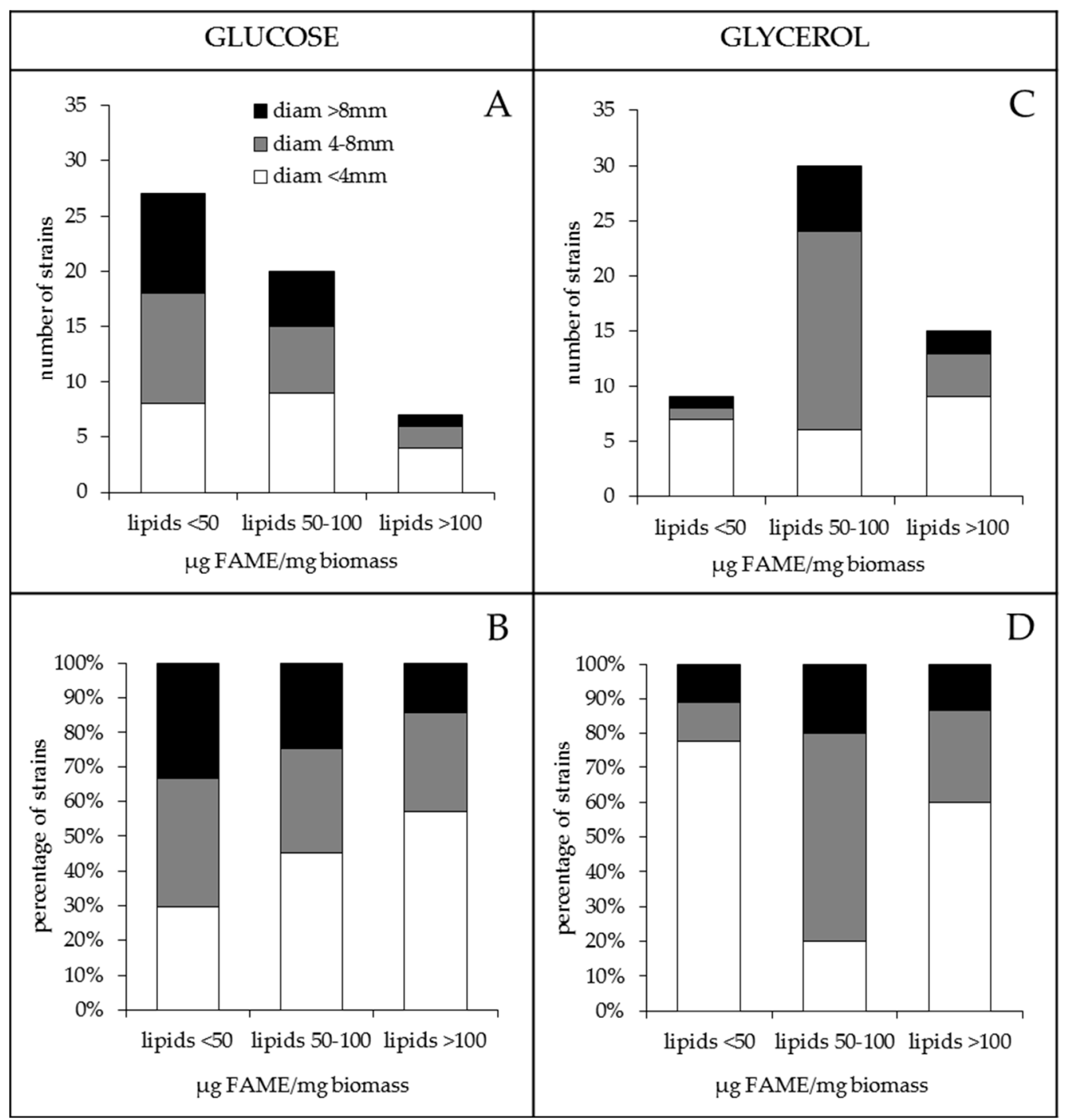

Figure 3. Classification of Streptomyces strains grown for $72 \mathrm{~h}$ at $28^{\circ} \mathrm{C}$ on solid modified R2YE limited in phosphate, according to low ( $<50 \mu \mathrm{g}$ FAME/mg biomass), medium (50-100 $\mu$ g FAME/mg biomass) or high (>100 $\mu \mathrm{g}$ FAME/mg biomass) total lipid/FAME content, on glucose (A,B) and glycerol (C,D), respectively. Each lipid class is represented by an histogram, and the numbers $(\mathbf{A}, \mathbf{B})$ or percentages $($ B,D) of strains with weak $(<4 \mathrm{~mm})$, medium $(4-8 \mathrm{~mm})$ and strong $(>8 \mathrm{~mm})$ antibiotic activity against Micrococcus luteus correspond to the white, grey and black parts of each histogram, respectively, on glucose (A,B) and glycerol (C,D).

Interestingly, on glucose, the proportion of strains yielding big halos of inhibition $(>8 \mathrm{~mm})$ decreases when the lipid content increases, and conversely, the proportion of strains yielding small 
halos of inhibition $(<4 \mathrm{~mm})$ increases when the lipid content increases (Figure 3b). These features, that are consistent with a negative correlation existing between lipid content and antibiotic activity, are not seen on glycerol (Figure $3 \mathrm{~d}$ ).

\subsection{A Statistically Significant Negative Pearson Correlation between Total Lipid Content and Antibiotic Activity Could Be Established with Some Exceptions}

In order to determine whether a statistically significant negative correlation between total lipid content and antibiotic activity could be established for glucose but also for glycerol, the lipid content of each strain was plotted against its respective antibiotic activity and a linear regression curve was established (Figure 4a,c). This indicated that on R2YE glucose or glycerol, no strong negative Pearson correlation ( $p$-value defined as $<0.05$ ) could be established between total lipid content and antibiotic activity since $p$-values of 0.135 and 0.227 were obtained for glucose and glycerol, respectively.

However, we noticed that if strains with extreme features were removed from our statistical analysis, a negative correlation between lipid content and antibiotic activity could be established. These strains were chosen by calculating quartiles. The graph (Figure $4 \mathrm{a}$ ), representing the fifty-four strains grown on glucose, was divided into quartiles according to their lipid content (dashed vertical lines) and antibiotic activity (dashed horizontal lines). Each quartile contains 25\% of each type of data. This delineated sixteen areas in which the strains had similar features concerning their lipid content and antibiotic activity. The three strains (S. brasiliensis, S. diastaticus subspecies diastaticus and S. albus) present in the lower corner area were in the quartiles bearing the lowest lipid content and no detectable antibiotic activity. By contrast, the three strains (S. bellus subspecies bellus, S. echinatus and $S$. aridus) present in upper corner area were in the quartiles bearing the highest lipid content and the highest antibiotic activity. The removal of these six strains with extreme features greatly improved the significance of the negative correlation between lipid content and antibiotic activity since statistically significant $p$-values of 0.002 and $0.013(<0.05)$ and Pearson correlation coefficients of -0.432 and -0.355 were then obtained for glucose- and glycerol-grown cultures, respectively (Figure $4 b, d)$.

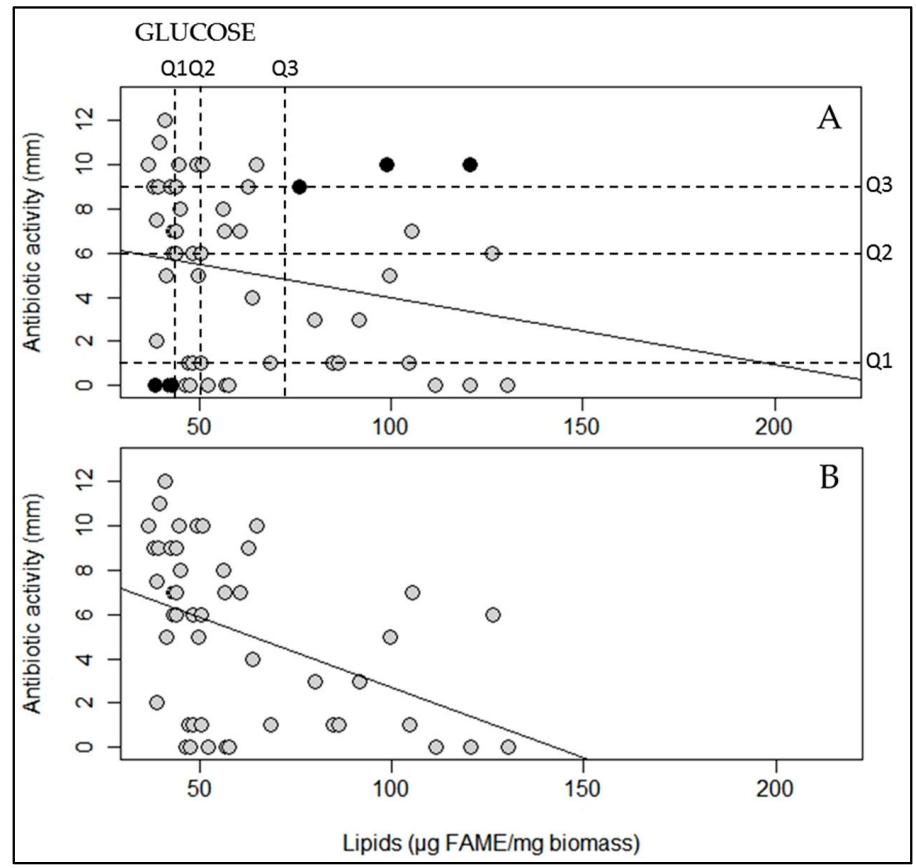

Figure 4. Cont. 


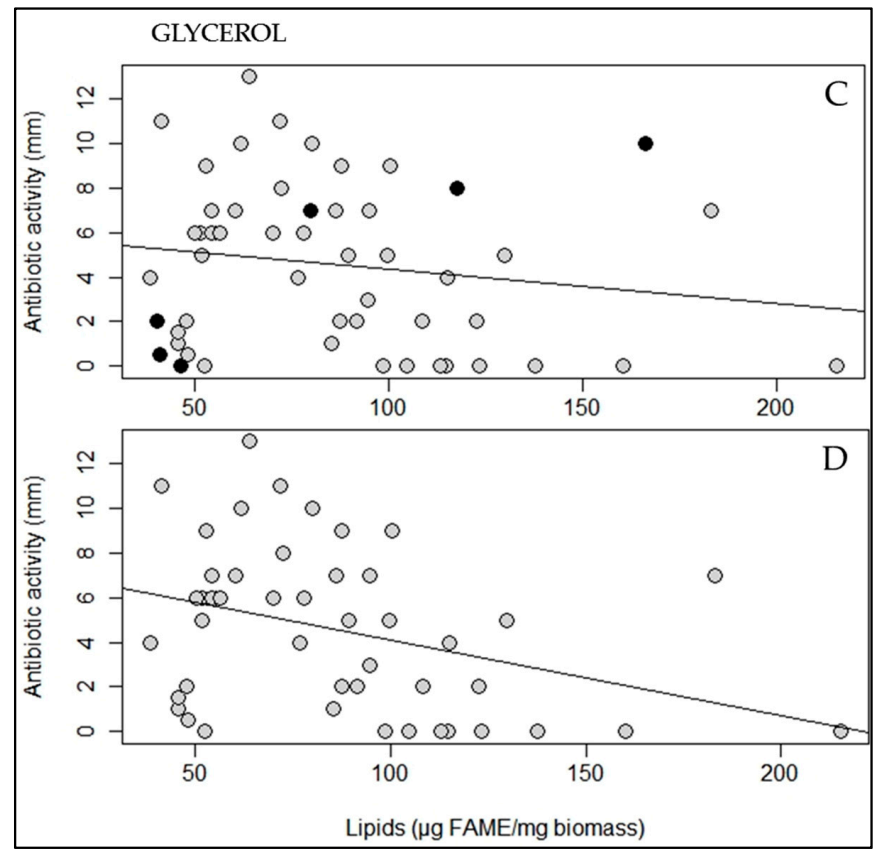

Figure 4. Lipid/FAME content of fifty-four Streptomyces strains grown on modified solid R2YE limited in phosphate for $72 \mathrm{~h}$ at $28^{\circ} \mathrm{C}$ with (A) $50 \mathrm{mM}$ glucose or (C) $100 \mathrm{mM}$ glycerol as the main carbon source was plotted against their antibiotic activity, and linear regression lines were calculated (black lines). In (A,C), FAME content and antibiotic activity did not show a statistically significant negative Pearson correlation (coefficients of -0.206 and $-0.167, p$-values of 0.135 and $0.227(>0.05)$, on glucose and glycerol, respectively). In an attempt to enhance the amplitude of this negative correlation, six strains constituting exceptions (black spots) were removed. These strains were chosen as described in the text, and the vertical and horizontal dashed lines represent the quartiles (Q1, Q2 and Q3) of lipid content and antibiotic activity, respectively. The lipid/FAME content of the forty-eight remaining strains grown in the presence of (B) $50 \mathrm{mM}$ glucose or (D) $100 \mathrm{mM}$ glycerol was plotted against their antibiotic activity, and linear regression lines were calculated (black lines). In (B,D), the FAME content and the antibiotic activity show a statistically significant negative Pearson correlation (coefficients of -0.432 and -0.355 , $p$-values of 0.002 and $0.013(<0.05)$, on glucose and glycerol, respectively).

2.3. Comparison of Streptomyces Antibioticus DSM 41,481 and DSM 40,868 Strains with Drastically Different Lipid Contents

In order to get a better understanding of the molecular basis for the huge difference in lipid content observed between strains, two strains named Streptomyces antibioticus DSM 41,481 (GenBank Acc. NZ_CM007717.1) and DSM 40,868 (ATCC 11,891, GenBank Acc. CP050504) that have among the highest and lowest lipid content, respectively, especially on glycerol, were studied in more detail.

The growth curves on R2YE glucose indicated that the strain DSM 41,481, bearing high lipid content, reached the stationary phase earlier and had a lower biomass yield than the strain DSM 40,868 (Figure 5a). The content in all lipid classes, and not only TAG as anticipated, was, on average, 2.5 fold higher in S. antibioticus DSM 41,481 than in DSM 40,868 (Figure 5c), consistent with FTIRS measurements [22-24]. Furthermore, the strain DSM 40,868 produced an antibiotic activity against Micrococcus luteus, whereas DSM 41,481 did not (Figure 5b). However, the production of antimycin by the strain DSM 41,481 was detected by LC/Corona-CAD (Figure 5d and Supplementary Figure S1), and the presence of an NRPS/PKS antimycin cluster in the genome of this strain was confirmed by anti-Smash [25] (Figure S2). In eukaryotes, antimycin impairs the correct functioning of the cytochrome c reductase, a key mitochondrial enzyme of the respiratory chain, and thus inhibits oxidative phosphorylation $[26,27]$ Streptomyces is known to possess several cytochrome c reductases/oxidases 
that could possibly be antimycin targets, but no report in the literature indicates that antimycin has an impact on the respiratory activity of Streptomyces producing strains. However, we think that it is likely that antimycin plays such a role in the producing strain since bio-active molecules often fulfill transient but important regulatory roles in their producer [12]. Furthermore, Streptomyces genomes are known to encode several eukaryotic-like enzymes as well as biosynthetic pathways directing the synthesis of molecules regulating their activity as well as that of their eukaryotic orthologs [28-30]. Since the strain DSM41481 stops growing earlier than the other strain, we cannot totally exclude that the antimycin produced by this strain alters the correct functioning of its oxidative metabolism, resulting in a reduction of TCA cycle activity. The latter would thus consume less acetylCoA, and an excess of acetylCoA would be stored as lipids.

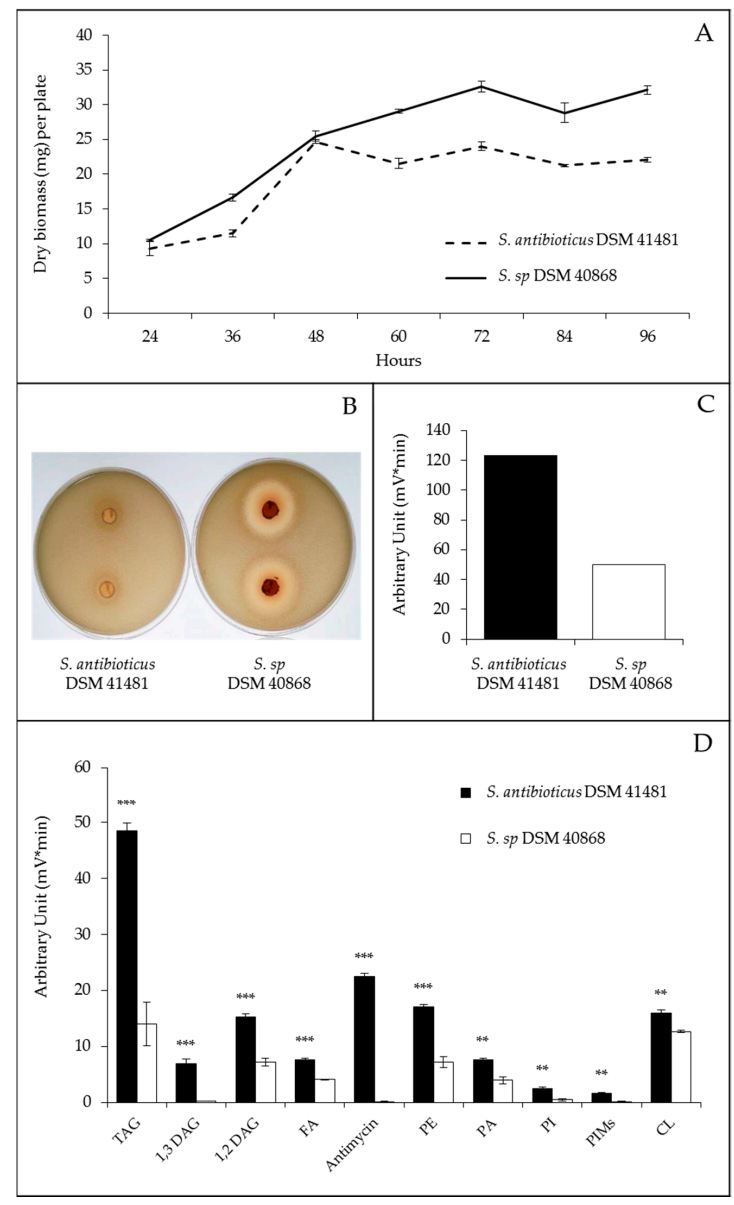

Figure 5. Cultures of S. antibioticus DSM 41,481 and S. sp. DSM 40,868 grown at $28{ }^{\circ} \mathrm{C}$ on solid R2YE limited in phosphate with glucose $(50 \mathrm{mM})$ as the main carbon source. (A) Growth curves of S. antibioticus DSM 41,481 (dotted lines) and S. sp. DSM 40,868 (continuous lines); (B) Pictures of the zones of inhibition of Micrococcus luteus growth around agar plugs of $72 \mathrm{~h}$-grown cultures of the Streptomyces strains; (C) Sum of all lipid classes (antimycin not included) in S. antibioticus DSM 41,481 (black histograms) and S. sp. DSM 40,868 (white histograms) according to LC/Corona-CAD data analysis; (D) Analysis of the total lipid contents in S. antibioticus DSM 41,481 (black histograms) and Streptomyces sp. DSM 40,868 (white histograms) grown for $72 \mathrm{~h}$ on solid R2YE glucose medium limited in phosphate according to LC/Corona-CAD. TAG stands for triacylglycerol; DAG, for diacylglycerol, FA for fatty acids; PE, for phosphatidylethanolamine; PA, for phosphatidic acid; PI, for phosphatidylinositol; PIMs, for phosphatidylinositol mannosides; and CL, for cardiolipid. Antimycin was detected in S. antibioticus DSM 41,481 but not in Streptomyces sp. DSM 40,868. Significant differences in lipid content between the two strains are represented by asterisks (ANOVA, ${ }^{* * *}=p<0.001 ;{ }^{* *}=p<0.01$ ). 
Since the two strains bear the same lipid classes, they most likely possess the same enzymes involved in the biosynthesis of the latter. As a consequence, the difference in their lipid content might be rather due to a difference in the availability of the necessary lipid precursor, acetylCoA. We thus examined, as a priority, through comparative genome analysis, genetic differences between the two strains in pathways involved in the generation, consumption or storage of acetylCoA that could be responsible for the high and low lipid contents of Streptomyces antibioticus DSM 41,481 and DSM 40,868, respectively.

\subsection{Comparative Analysis of the Sequence of the Genome of Streptomyces Antibioticus DSM 41,481 and DSM 40,868}

The genomes of the two strains of S. antibioticus-DSM 41,481 and DSM 40,868, called OSCP and OSBF in our collection-were fully sequenced thanks to a combination of Nanopore (Oxford Nanopore Technologies) and Illumina technologies. A hybrid assembly was generated to give a single contig per genome, of 8,473,575 bp and 9,195,693 bp for DSM 41,481 and DSM 40,868, respectively. They possess Terminal Inverted Repeats (TIR) of $26,378 \mathrm{bp}$ and $13,483 \mathrm{bp}$, respectively. The genomic sequence of strain DSM 41,481 was almost identical to that present in the Genbank database (CM007717.1), with a very strong nucleotide identity over the whole genome (greater than $99.9 \%$ ). Moreover, the two genomic sequences are fully collinear throughout (not shown). Due to the high quality of the sequencing performed in this work thanks to the dual technology used, we have deposited it in Genbank under the accession number CP050692.

In addition, the assignment of the strain DSM 40,868 to the species S. antibioticus was questioned by phylogenetic analyses carried out using the acquired genomic sequence. Indeed, its $16 S$ rDNA sequence was identical to that of $S$. lavenduligriseus strain NBRC 13,405, while it bears only $96.47 \%$ identity with S. antibioticus DSM 41,481. Furthermore, a phylogenetic reconstruction showed that DSM 40,868 better clustered with $S$. lavenduligriseus strain NBRC 13,405 than with $S$. antibioticus strains. However, since DSM 40,868 is listed as S. antibioticus in both the DSMZ and ATCC website collections (DSM 40,868 is also registered as ATCC 11,891), re-naming this strain as S. lavenduligriseus would be confusing, so this strain was called S. sp. DSM 40,868, and its genome sequence was registered under the Genbank accession number CP050504. The annotation was performed using the RAST tool kit (RASTtk) [31], available on the Rapid Annotation using Subsystem Technology (RAST) platform. S. antibioticus DSM 41,481 included 7823 predicted coding DNA sequences (CDSs), while Streptomyces sp. DSM 40,868 contained 8637 CDSs. AntiSMASH was used to predict the presence of biosynthetic gene clusters [25]. Twenty-six and forty-six gene clusters were found in the genomes of S. antibioticus DSM 41,481 and S. sp. DSM 40,868, respectively (Figures S2-S4).

In the comparative analysis of the two genomes, we chose to consider only the reactions catalyzed by a single enzyme that was present in one species but absent in the other.

We first noticed that the enzyme 3.1.3.10 that catalyzes the dephosphorylation of glucose $1 \mathrm{P}$ to glucose does not exist in the high lipid-containing strain, S. antibioticus DSM 41,481 (Figure S5). The absence of this enzyme might eliminate a futile ATP-consuming cycle of the dephosphorylation/re-phosphorylation of glucose and would allow a more direct and efficient conversion of Glc1P to Glc6P, favorable for the generation of acetylCoA (Figure S5).

The presence in the strain DSM 41,481, but not in DSM 40,868, of the enzyme 1.1.1.83 that catalyzes the conversion of malate into pyruvate and of 2.3.1.54 (Figure S6) that catalyzes the conversion of pyruvate into acetylCoA (Figures S6 and S7) is likely to have a positive impact on acetylCoA availability and thus on the lipid content of DSM 41,481. Furthermore, two enzymes (1.1.99.2 and 2.8.3.12) converting 2-oxoglutarate into hydroxyglutarylCoA are present in the strain DSM 41,481 but absent in DSM 40,868 (Figure S6). Since hydroxyglutarylCoA can eventually lead to the synthesis of crotomyl and butanoylCoA, precursors of odd chain or branched fatty acids, its generation might thus have a positive impact on lipid content. 
Concerning nitrogen metabolism, we noted the presence in DSM 40,868 but not in DSM 41,481 of a cyanate lyase (4.2.1.104) that catalyzes the reaction of cyanate with bicarbonate, yielding carbon dioxide and $\mathrm{NH}_{4}$ (Figure S8). By contrast, we noted the presence in DSM 41,481 but not in DSM 40,868 of the enzyme 1.4.1.3 (Figure S9) that catalyzes the conversion of oxoglutarate into glutamate, consuming a molecule of $\mathrm{NH}_{4}$. Since $\mathrm{NH}_{4}$ is known to be inhibitory to lipid biosynthesis [32-34], its generation via the cyanate lyase in DSM 40,868 and its consumption, for glutamate generation, in DSM 41,481 might have negative and positive impacts on lipid accumulation in DSM 40,868 and DSM 41,481, respectively.

We also noticed that one of the multiple routes (catalyzed by the enzyme 1.1.1.169) involved in the conversion of 2-dehydropentoate into pentoate, a metabolite used for the biosynthesis of coenzyme A, is present in DSM 41,481 but absent in DSM 40,868 (Figure S10). Since coenzyme A is crucial to activate acetylCoA for fatty acid biosynthesis, the absence of this enzyme in DSM 40,868 might limit coenzyme A biosynthesis, contributing to its low lipid content.

Altogether, these genetic differences are consistent with the high and low lipid contents of $S$. antibioticus DSM 41,481 and Streptomyces sp. DSM 40,868, respectively. However, one cannot totally exclude that the main reason for the high lipid content of S. antibioticus DSM 41,481 is its production of antimycin as mentioned above.

\section{Discussion}

In this issue, we tried to determine whether a statistically significant negative Pearson correlation could be established between total lipid content and antibiotic activity in Streptomyces species, since previous studies suggested that such a correlation might exist [7]. The lipid content of a cell relies on its ability to synthetize glycerol3P and acetylCoA, which both stem mainly (but not exclusively) from glycolysis. AcetylCoA is a metabolic node that can have various fates. During active growth, it constitutes the main fuel of the TCA cycle, which generates the metabolic precursors of amino acids used for protein biosynthesis or other anabolic processes. However, if, for some reason (such as nitrogen or phosphate limitation), the TCA cycle and thus anabolic processes slow down, acetylCoA can then be stored as lipids, as in S. lividans, or used for polyketide antibiotics biosynthesis, as in S. coelicolor. In such a context of low anabolism, amino acids generated from intermediates of the TCA cycle can also be used for the biosynthesis of peptide antibiotics. Since the biosynthesis of these bioactive molecules directly or indirectly requires acetyl $\mathrm{CoA}$, their synthesis might be in direct competition with that of lipids. This could explain the negative correlation existing between these two processes, and these molecules can be seen as metabolic sinks when, in conditions of growth slowdown, the metabolites generated by anabolism exceed the needs of anabolism.

However, things might not be as simple as described above, since the biosynthesis of bioactive molecules is usually triggered by some specific physiological or environmental conditions, often linked to nutritional limitation and growth slowdown [12,35], and these molecules are believed to fulfill important regulatory roles for the producing bacteria in such contexts [12]. For instance, S. coelicolor, a strain characterized by an active oxidative metabolism consuming acetylCoA and thus bearing a low lipid content, produces antibiotics proposed to contribute to the regulation of its energetic metabolism [12]. Our data suggested that the existence of a negative correlation between lipid content and antibiotic activity, previously established for S. coelicolor, was also true for most Streptomyces strains, at least on R2YE glucose. However, the existence of exceptions to this general rule was noted. These strains either bear a low lipid content and undetectable antibiotic activity (Type I: S. brasiliensis, S. diastaticus subspecies diastaticus and S. albus) or a high lipid content and high antibiotic activity (Type II: S. bellus subspecies bellus, S. echinatus and S. aridus). One should be aware that assessing antibiotic activity via the measurement of the diameter of the growth inhibition zones of Micrococcus luteus might not reveal all antibiotic production. Indeed, the existence of Type I strains could be easily explained by the insensitivity of Micrococcus luteus to the antibiotic produced, which is thus not detected. This was exemplified by the production of antimycin by DSM41481, which has no impact on Micrococcus growth. By contrast, Type II strains may produce very small amounts of a specific 
antibiotic that Micrococcus luteus is highly sensitive to or produce low levels of multiple antibiotics that synergistically impact Micrococcus luteus growth etc. Anyhow, if antibiotic biosynthesis is triggered in a strain with a low to medium lipid content, antibiotic biosynthesis is likely to have a significant impact on its lipid content, but such an impact will be hardly visible in a strain bearing high lipid content.

Furthermore, to explain the unexpected lower antibiotic activity on glycerol than on glucose, we propose that oxidative stress might be less severe on glycerol than on glucose since the catabolism of glycerol-and more precisely its conversion into dihydroxyacetone by a glycerol dehydrogenase or into glyceraldehyde by a glyceraldehyde reductase-generates more NADPH than the catabolism of glucose [36,37]. NADPH is an indispensable reduced co-factor necessary to combat and thus reduce oxidative stress [38], since some reports in the literature mention that the biosynthesis of specific antibiotics might be triggered by oxidative stress [39-43]. The biosynthesis of such antibiotics is thus expected to be reduced on glycerol. Antibiotics of this class (defined as class II in Virolle, 2020 [12]) are thought to have anti-oxidant functions via their ability to capture electrons from the respiratory chain to prevent the formation of ROS or NOS, but in doing so, they would also be inhibitory to respiration and thus toxic for living cells.

Lastly, the comparative genomic analysis of two strains-both named Streptomyces antibioticus, DSM 41,481 and DSM 40,868-that we found to be more phylogenetically related to Streptomyces lavenduligriseus indicated that some genetic differences in various pathways related to the generation/consumption of acetylCoA could be responsible for such differences.

\section{Materials and Methods}

\subsection{Bacterial Strains, Media and Growth Conditions}

Most of the fifty-four strains used in this study were ATCC/NRLL/DSM strains. The few that do not come from these collections (S. albus, S. spectabilis, S. actuosus, S. coelicolor M145, S. lividans TK24, S. pristinaespiralis and S. rimosus 2535) were generous gifts from John Innes Institute. Viable spores $\left(10^{6}\right)$ of each strain were plated on the surface of four petri dishes (42 $\mathrm{mm}$ diameter) of solid modified R2YE medium [44] covered by a cellophane disk (Focus Packaging \& Design Ltd., Louth, UK). No sucrose and no $\mathrm{K}_{2} \mathrm{HPO}_{4}$ were added to this modified solid R2YE medium supplemented with either glucose $(50 \mathrm{mM})$ or glycerol $(100 \mathrm{mM})$ as the main carbon sources. However, the determination of the content of free phosphate in this medium with a PiBlue phosphate assay kit (Gentaur, France) revealed a concentration of free $\mathrm{Pi}$ of $1 \mathrm{mM}$, corresponding to a situation of phosphate limitation. The plates were incubated for $72 \mathrm{~h}$ at $28{ }^{\circ} \mathrm{C}$. After $72 \mathrm{~h}$ of incubation, mycelia were scraped off the cellophane disks of each plate with a spatula and lyophilized in order to assess their total lipid contents by Fourier transform infra- red spectroscopy (FTIRS) [22-24]. Four agar plugs of each plate were deposited on a lawn of the Actinobacteria Micrococcus luteus in order to determine the antibiotic activity of the Streptomyces strains against this strain that is known to be sensitive to most antibiotics $[45,46]$. The growth curves of Streptomyces antibioticus DSM 41,481 and Streptomyces sp. DSM 40,868, the two strains with extremely high and low total lipid contents, were obtained on the same R2YE medium (Figure 5a), and their lipid content was assessed by LC/Corona-CAD (Figure 5d).

\subsection{Determination of Total Lipid Content Using Attenuated Total Reflectance-Fourier Transform Infra Red Spectroscopy (ATR-FTIRS) Measurements}

In order to determine total lipid content, lyophilized mycelial samples of the Streptomyces strains were subjected to FTIR spectroscopy using a Bruker Vertex 70 FTIR spectrophotometer with a diamond ATR attachment (PIKE MIRacle crystal plate diamond ZnSe) and an MCT detector with a liquid nitrogen cooling system. A reference spectrum resulting from one hundred averaged scans obtained in absence of any sample on the Infra Red support was acquired before each sample analysis. Scans were conducted from $3600 \mathrm{~cm}^{-1}$ to $600 \mathrm{~cm}^{-1}$ with a spectral resolution of $4 \mathrm{~cm}^{-1}$, with 100 averaged scans for each sample. This technique allows the establishment of spectral fingerprints of the complex biological 
structures under investigation. The $\mathrm{C}-\mathrm{H}$ stretching bands of the $\mathrm{CH}_{2}$ of fatty acid chains (between 2959 and $2852 \mathrm{~cm}^{-1}$ ) and the $\mathrm{C}=\mathrm{O}$ ester stretching band of the ester carbonyl (band near $1740 \mathrm{~cm}^{-1}$ ) are characteristic of lipids including mainly polar membrane lipids and neutral lipids such as TAG. The height of the sharp and distinct $\mathrm{C}=\mathrm{O}$ ester stretching band of the ester carbonyl is especially relevant for monitoring the total intracellular lipid contents of the strains [23]. Furthermore, since the biomass protein content can be directly characterized by the amplitude of the Amide I absorption band $\left(1650 \mathrm{~cm}^{-1}\right)$, all the FTIR spectra can be normalized to this band, allowing the comparison of the total lipid contents of the mycelial lawns of different strains. The total lipid contents of the strains assessed by FTIRS, expressed as arbitrary units, was converted into $\mu \mathrm{g}$ of Fatty Methyl Esters (FAME) per mg of dry mycelium using the converting equation established by Millan-Oropeza et al. (2017) [24]. Indeed, in the presence of methanol, fatty acids constitutive of all cellular lipids are esterified to yield FAME that can be accurately quantified by GC/MS [22-24]. The differences in the lipid contents of the strains were clear and highly reproducible.

\subsection{Determination of the Antibiotic Activity of the Strains against Micrococcus Luteus}

In order to determine the inhibitory impact that each Streptomyces strain had on Micrococcus luteus growth, four agar cylinders were taken aseptically from each of the replica plates using an appropriate device and were deposited onto the surface of LB agar plates, prepared as follows. Twenty-five microliters of a fresh culture of Micrococcus luteus (OD $600 \mathrm{~nm}$ of 0.4) were added to $3 \mathrm{~mL}$ of soft nutrient agar (SNA) that was poured onto the surface of the LB agar plate. As soon as the SNA containing Micrococcus luteus was solidified, the agar plugs were deposited on the surface of the plates and incubated $24 \mathrm{~h}$ at $37^{\circ} \mathrm{C}$. For each plate, the size of the four zones of inhibition was measured and reported in an Excel file, and the mean was calculated.

\subsection{Statistical Analysis of the Correlation between the Lipid Content and Antibiotic Activity of Studied Streptomyces Strains}

The total lipid contents of the strains were expressed as mean \pm standard deviation in bar graphs and were subjected to the ANOVA test (Figure 1a,b). Correlation analyses between lipid content and antibiotic activity were achieved using Pearson correlation (Figure 4). All statistical methods and data representation were conducted in R 3.3.2 [47], using the "RVAideMemoire" package [48]; a $p$-value $<0.05$ was considered as statistically significant.

\subsection{Lipid Extraction and Characterization by LC/Corona-CAD and LC/MS}

Lipid extraction was performed by a procedure derived from Folch's method [49] from four independent cultures of Streptomyces antibioticus DSM 41,481 and DSM 40,868. A defined volume (4.5 mL) of chloroform/methanol (1:2) was added to $10 \mathrm{mg}$ of lyophilized Streptomyces mycelium and vortexed for $30 \mathrm{~s}$. The mixture was left at ambient temperature for 1 hour, then $1.25 \mathrm{~mL}$ of water was added, and the mixture was vortexed for $30 \mathrm{~s}$. The mixture was then centrifuged $(1000 \times g$ for $10 \mathrm{~min}$ ) to obtain phase separation. The lower organic phase was collected, and the upper aqueous phase was submitted to a second extraction by adding $2 \mathrm{~mL}$ of chloroform/methanol (85:15). The two organic phases were pooled and evaporated under a stream of nitrogen at room temperature. The dry residue was dissolved in $400 \mu \mathrm{L}$ of isooctane/chloroform (4:1) before analysis. The chromatographic conditions have been described previously [50]. Briefly, lipid class analysis was performed with an Inertsil Silica ( $150 \mathrm{~mm} \times 2.1 \mathrm{~mm}$ I.D, $5 \mu \mathrm{m}$ ) column (GL Sciences Inc., Tokyo, Japan) thermostated at $40{ }^{\circ} \mathrm{C}$. The HPLC instrumentation consisted of the system Dionex U-3000 RSLC (Thermofisher, Villebon, France). A quaternary solvent gradient (Supplementary Table S1) was used to elute all the lipid classes present in the sample by increasing the order of polarity. Lipid class identification was verified by coupling the chromatographic separation to mass spectrometry. MS analyses were performed with a LTQ-Orbitrap Velos Pro (Thermo Fisher Scientific) equipped with an APPI ion source. The $\mathrm{MS}^{2}$ and $\mathrm{MS}^{3}$ spectra were obtained in data-dependent acquisition (DDA) 
mode. Lipid detection was performed using a Corona-CAD system (ESA, Chelmsford, MA, USA) [50]; the signal was acquired with a Chromeleon data station (Thermo Fisher Scientific, Villebon-sur-Yvette, France). Corona-CAD is a universal detector used for liquid chromatography and described in Dixon and Peterson [51]. The differences in the composition of the lipid classes in the samples are expressed as peak areas. All the data were subjected to Student's t-test using R 3.3.2 [47] and the "multcompView" package [52]. The results obtained are presented as the mean \pm standard error; a $p$-value $<0.05$ was considered as statistically significant.

\subsection{DNA Isolation, Genome Sequencing and Annotation}

After growth in liquid Hickey-Tresner medium [6] at $30{ }^{\circ} \mathrm{C}$ for $30 \mathrm{~h}$, DNA purification was performed using the salting-out method [6], followed by chloroform extraction. A hybrid assembly using Oxford Nanopore technology for scaffolding and Illumina technology for sequence improvement was performed. Nanopore reads were generated on a gridION system. The coverage was $30 \times$ and $67 \times$ for strains DSM 41,481 and DSM 40,868, respectively. The Illumina paired-end libraries were sequenced using a MiSeq system (Illumina). The coverages of the paired-end reads (length, $301 \mathrm{bp}$ ) ranged from $249 \times$ to $312 \times$. The hybrid assembly was performed using Unicycler [53]. The accession numbers are CP050692 and CP050504 for S. antibioticus DSM 41,481 and Streptomyces sp. DSM 40,868, respectively. Sequencing and assembly were performed via the I2BC NGS platform (France). The automatic annotation of the genome sequences was achieved using the RAST tool kit (RASTtk [31]) available on the Rapid Annotation using Subsystem Technology (RAST) platform. Furthermore, the annotation files were used as the input for antiSMASH [25] in order to predict the biosynthetic gene clusters content of each genome. Biosynthetic pathways were mapped with KEGG, using the GHOSTZ search program and the BBH (bi-directional best hit) assignment method [54]. We used the default gene data set, to which we added the data sets available for five Streptomyces species (Streptomyces coelicolor, Streptomyces avermitilis, Streptomyces griseus, Streptomyces scabiei and Streptomyces noursei). The presence/absence of specific enzymatic steps was manually checked using BLAST searches [55].

Supplementary Materials: The following are available online at http://www.mdpi.com/2079-6382/9/6/280/s1, Figure S1: LC/Corona-CAD results of total lipid content in Streptomcyces antibioticus DSM 41,481 and Streptomyces sp. DSM 40,868 grown for $72 \mathrm{~h}$ on solid R2YE glucose medium limited in phosphate, Figure S2: Anti-Smash analysis of the genome of Streptomyces antibioticus DSM 41,481 (8,473,575 bp), Figure S3: Anti-smash analysis of the genome of Streptomyces sp. DSM 40,868 (registered in gene bank as Streptomyces antibioticus DSM 40,868 or ATCC 11,891) $(9,195,693 \mathrm{bp})$, Figure S4: Chromosomal position of the biosynthetic gene clusters predicted by antiSMASH along the chromosome of Streptomyces sp. DSM 40,868 and Streptomyces antibioticus DSM 41,481. Metabolites synthetized by similar biosynthetic pathways are signaled by a vertical bar and named, Figure S5: Comparative analysis of glycolysis/gluconeogenesis pathway of DSM 40,868 and DSM 41,481, Figure S6: Comparative analysis of butanoate metabolism of DSM 40,868 and DSM 41,481, Figure S7: Comparative analysis of pyruvate metabolism of DSM 40,868 and DSM 41,481, Figure S8: Comparative analysis of nitrogen metabolism of DSM 40,868 and DSM 41,481, Figure S9: Comparative analysis of glutamine/glutamate metabolism of DSM 40,868 and DSM 41,481, Figure S10: Comparative analysis of pantothenate and CoA biosynthetic pathways of DSM 40,868 and DSM 41,481, Table S1: Quaternary gradient mobile phase composition.

Author Contributions: Conceptualization, M.-J.V.; methodology, M.D., S.A., A.T. and P.L.; validation, M.-J.V., C.L., S.A., P.C., A.T. and P.L.; formal analysis, C.L. and A.T.; investigation, M.D., C.L., S.A., A.T. and P.L.; resources, M.D., S.A., P.C., A.T. and P.L.; writing-original draft preparation, M.-J.V.; writing-review and editing, C.L., S.A., P.C., A.T. and P.L.; visualization, C.L. and A.T.; supervision, M.-J.V.; project administration, M.-J.V.; funding acquisition, M.-J.V. All authors have read and agreed to the published version of the manuscript.

Funding: This research was funded by dotation of the "Centre National de la Recherche Scientifique" CNRS (http://www.cnrs.fr) and University Paris Saclay (https://www.universite-paris-saclay.fr/en) as well as by the Investment for the Future "Biotechnologies et Bioressources" n¹1-BTBR-0003 (PROBIO3), the ANR BioSound-IR (ANR-15-CE09-0002) and Innovantibio (ANR-17-ASTR-0018).

Acknowledgments: We thank Ariane Deniset-Besseau and Rolando Rebois (University of Paris-Saclay) for training and helping Michelle David to assess total lipid content of Streptomyces strains using "Attenuated Total Reflectance-Fourier Transform Infra Red Spectroscopy" (ATR-FTIRS).

Conflicts of Interest: The authors declare no conflict of interest. 


\section{References}

1. Pham, J.V.; Yilma, M.A.; Feliz, A.; Majid, M.T.; Maffetone, N.; Walker, J.R.; Kim, E.; Cho, H.J.; Reynolds, J.M.; Song, M.C.; et al. A Review of the microbial production of bioactive natural products and biologics. Front. Microbiol. 2019, 10, 1404. [CrossRef]

2. Barka, E.A.; Vatsa, P.; Sanchez, L.; Gaveau-Vaillant, N.; Jacquard, C.; Klenk, H.-P.; Clément, C.; Ouhdouch, Y.; van Wezel, G.P. Taxonomy, physiology, and natural products of actinobacteria. Microbiol. Mol. Biol. Rev. 2016, 80, 1-43. [CrossRef]

3. Chater, K.F. Recent advances in understanding Streptomyces. F1000Research 2016, 5, 2795. [CrossRef]

4. Van Wezel, G.P.; McDowall, K.J. The regulation of the secondary metabolism of Streptomyces: New links and experimental advances. Nat. Prod. Rep. 2011, 28, 1311. [CrossRef]

5. Alvarez, H.M.; Steinbuchel, A. Triacylglycerols in prokaryotic microorganisms. Appl. Microbiol. Biotechnol. 2002, 60, 367-376. [CrossRef] [PubMed]

6. Kieser, T.; Bibb, M.J.; Buttner, M.J.; Chater, K.F.; Hopwood, D.A. Practical Streptomyces Genetics; Innes: Norwich, UK, 2000; ISBN 978-0-7084-0623-6.

7. Esnault, C.; Dulermo, T.; Smirnov, A.; Askora, A.; David, M.; Deniset-Besseau, A.; Holland, I.-B.; Virolle, M.-J. Strong antibiotic production is correlated with highly active oxidative metabolism in Streptomyces coelicolor M145. Sci. Rep. 2017, 7, 200. [CrossRef] [PubMed]

8. Le Maréchal, P.; Decottignies, P.; Marchand, C.H.; Degrouard, J.; Jaillard, D.; Dulermo, T.; Froissard, M.; Smirnov, A.; Chapuis, V.; Virolle, M.-J.; et al. Comparative proteomic analysis of Streptomyces lividans Wild-Type and ppk mutant strains reveals the importance of storage lipids for antibiotic biosynthesis. Appl. Environ. Microbiol. 2013, 79, 5907-5917. [CrossRef] [PubMed]

9. Millan-Oropeza, A.; Henry, C.; Lejeune, C.; David, M.; Virolle, M.-J. Expression of genes of the Pho regulon is altered in S. coelicolor. Sci. Rep. 2020, 10, 8492. [CrossRef] [PubMed]

10. Chouayekh, H.; Virolle, M.J. The polyphosphate kinase plays a negative role in the control of antibiotic production in Streptomyces lividans. Mol. Microbiol. 2002, 43, 919-930. [CrossRef] [PubMed]

11. Ghorbel, S. Regulation of ppk expression and in vivo function of Ppk in Streptomyces lividans TK24. J. Bacteriol. 2006, 188, 6269-6276. [CrossRef]

12. Virolle, M.-J. A challenging view: Antibiotics play a role in the regulation of the energetic metabolism of the producing bacteria. Antibiotics 2020, 9, 83. [CrossRef] [PubMed]

13. Tenconi, E.; Traxler, M.F.; Hoebreck, C.; Wezel, G.P.; Rigali, S. Production of prodiginines is part of a programmed cell death process in streptomyces coelicolor. Front. Microbiol. 2018, 9, 1742. [CrossRef] [PubMed]

14. Olukoshi, E.R.; Packter, N.M. Importance of stored triacylglycerols in Streptomyces: Possible carbon source for antibiotics. Microbiology 1994, 140, 931-943. [CrossRef] [PubMed]

15. Packter, N.M.; Olukoshi, E.R. Ultrastructural studies of neutral lipid localisation in Streptomyces. Arch. Microbiol. 1995, 164, 420-427. [CrossRef] [PubMed]

16. Foley, T.L.; Young, B.S.; Burkart, M.D. Phosphopantetheinyl transferase inhibition and secondary metabolism: PPTase inhibition and secondary metabolism. FEBS J. 2009, 276, 7134-7145. [CrossRef] [PubMed]

17. Craney, A.; Ozimok, C.; Pimentel-Elardo, S.M.; Capretta, A.; Nodwell, J.R. Chemical perturbation of secondary metabolism demonstrates important links to primary metabolism. Chem. Biol. 2012, 19, 1020-1027. [CrossRef] [PubMed]

18. Banchio, C.; Gramajo, H. A stationary-phase acyl-coenzyme A synthetase of streptomyces coelicolor A3(2) is necessary for the normal onset of antibiotic production. Appl. Environ. Microbiol. 2002, 68, 4240-4246. [CrossRef]

19. Wang, W.; Li, S.; Li, Z.; Zhang, J.; Fan, K.; Tan, G.; Ai, G.; Lam, S.M.; Shui, G.; Yang, Z.; et al. Harnessing the intracellular triacylglycerols for titer improvement of polyketides in Streptomyces. Nat. Biotechnol. 2020, 38, 76-83. [CrossRef]

20. Chen, Y.; Metz, J.; Miller-Xavier, R.K.; Wang, G. Unlocking a new target for streptomycetes strain improvement. Synth. Syst. Biotechnol. 2020, 5, 33-34. [CrossRef]

21. Schauner, C.; Dary, A.; Lebrihi, A.; Leblond, P.; Decaris, B.; Germain, P. Modulation of lipid metabolism and spiramycin biosynthesis in Streptomyces ambofaciens unstable mutants. Appl. Environ. Microbiol. 1999, 65, 2730-2737. [CrossRef] 
22. Vitry, P.; Rebois, R.; Bourillot, E.; Deniset-Besseau, A.; Virolle, M.-J.; Lesniewska, E.; Dazzi, A. Combining infrared and mode synthesizing atomic force microscopy: Application to the study of lipid vesicles inside Streptomyces bacteria. Nano Res. 2016, 9, 1674-1681. [CrossRef]

23. Deniset-Besseau, A.; Prater, C.B.; Virolle, M.-J.; Dazzi, A. Monitoring TriAcylGlycerols accumulation by atomic force microscopy based infrared spectroscopy in streptomyces species for biodiesel applications. J. Phys. Chem. Lett. 2014, 5, 654-658. [CrossRef] [PubMed]

24. Millan-Oropeza, A.; Rebois, R.; David, M.; Moussa, F.; Dazzi, A.; Bleton, J.; Virolle, M.-J.; Deniset-Besseau, A. Attenuated total reflection fourier transform infrared (ATR FT-IR) for rapid determination of microbial cell lipid content: Correlation with gas chromatography-mass spectrometry (GC-MS). Appl. Spectrosc. 2017, 71, 2344-2352. [CrossRef] [PubMed]

25. Blin, K.; Wolf, T.; Chevrette, M.G.; Lu, X.; Schwalen, C.J.; Kautsar, S.A.; Suarez Duran, H.G.; de Los Santos, E.L.C.; Kim, H.U.; Nave, M.; et al. AntiSMASH 4.0-improvements in chemistry prediction and gene cluster boundary identification. Nucleic Acids Res. 2017, 45, W36-W41. [CrossRef]

26. Seipke, R.F.; Hutchings, M.I. The regulation and biosynthesis of antimycins. Beilstein J. Org. Chem. 2013, 9, 2556-2563. [CrossRef]

27. Joynt, R.; Seipke, R.F. A phylogenetic and evolutionary analysis of antimycin biosynthesis. Microbiology (Reading, England) 2018, 164, 28-39. [CrossRef]

28. Shanbhag, P.; Bhave, S.; Vartak, A.; Kulkarni-Almeida, A.; Mahajan, G.; Villanueva, I.; Davies, J. Screening of microbial extracts for anticancer compounds using streptomyces kinase inhibitor assay. Nat. Prod. Commun. 2015, 10, 1287-1291. [CrossRef]

29. Chevrette, M.G.; Gutiérrez-García, K.; Selem-Mojica, N.; Aguilar-Martínez, C.; Yañez-Olvera, A.; Ramos-Aboites, H.E.; Hoskisson, P.A.; Barona-Gómez, F. Evolutionary dynamics of natural product biosynthesis in bacteria. Nat. Prod. Rep. 2020, 37, 566-599. [CrossRef]

30. Yagüe, P.; Lopez-Garcia, M.T.; Rioseras, B.; Sanchez, J.; Manteca, A. New insights on the development of Streptomyces and their relationships with secondary metabolite production. Curr. Trends Microbiol. 2012, 8, 65-73.

31. Brettin, T.; Davis, J.J.; Disz, T.; Edwards, R.A.; Gerdes, S.; Olsen, G.J.; Olson, R.; Overbeek, R.; Parrello, B.; Pusch, G.D.; et al. RASTtk: A modular and extensible implementation of the RAST algorithm for building custom annotation pipelines and annotating batches of genomes. Sci. Rep. 2015, 5, 8365. [CrossRef]

32. Valledor, L.; Furuhashi, T.; Recuenco-Muñoz, L.; Wienkoop, S.; Weckwerth, W. System-level network analysis of nitrogen starvation and recovery in Chlamydomonas reinhardtii reveals potential new targets for increased lipid accumulation. Biotechnol. Biofuels 2014, 7, 171. [CrossRef] [PubMed]

33. Goncalves, E.C.; Koh, J.; Zhu, N.; Yoo, M.-J.; Chen, S.; Matsuo, T.; Johnson, J.V.; Rathinasabapathi, B. Nitrogen starvation-induced accumulation of triacylglycerol in the green algae: Evidence for a role for ROC40, a transcription factor involved in circadian rhythm. Plant J. 2016, 85, 743-757. [CrossRef] [PubMed]

34. Morin, N.; Cescut, J.; Beopoulos, A.; Lelandais, G.; Le Berre, V.; Uribelarrea, J.-L.; Molina-Jouve, C.; Nicaud, J.-M. Transcriptomic analyses during the transition from biomass production to lipid accumulation in the oleaginous yeast Yarrowia lipolytica. PLoS ONE 2011, 6, e27966. [CrossRef] [PubMed]

35. Liu, G.; Chater, K.F.; Chandra, G.; Niu, G.; Tan, H. Molecular regulation of antibiotic biosynthesis in streptomyces. Microbiol. Mol. Biol. Rev. 2013, 77, 112-143. [CrossRef]

36. Husain, A.; Sato, D.; Jeelani, G.; Soga, T.; Nozaki, T. Dramatic increase in glycerol biosynthesis upon oxidative stress in the anaerobic protozoan parasite Entamoeba histolytica. PLoS Negl. Trop. Dis. 2012, 6, e1831. [CrossRef]

37. Pahlman, A.K.; Granath, K.; Ansell, R.; Hohmann, S.; Adler, L. The yeast glycerol 3-phosphatases Gpp1p and Gpp2p are required for glycerol biosynthesis and differentially involved in the cellular responses to osmotic, anaerobic, and oxidative stress. J. Biol. Chem. 2001, 276, 3555-3563. [CrossRef]

38. Agledal, L.; Niere, M.; Ziegler, M. The phosphate makes a difference: Cellular functions of NADP. Redox Rep. 2010, 15, 2-10. [CrossRef]

39. Beites, T.; Oliveira, P.; Rioseras, B.; Pires, S.D.S.; Oliveira, R.; Tamagnini, P.; Moradas-Ferreira, P.; Manteca, Á.; Mendes, M.V. Streptomyces natalensis programmed cell death and morphological differentiation are dependent on oxidative stress. Sci. Rep. 2015, 5, 12887. [CrossRef] 
40. Beites, T.; Pires, S.D.S.; Santos, C.L.; Osório, H.; Moradas-Ferreira, P.; Mendes, M.V. Crosstalk between ROS homeostasis and secondary metabolism in S. natalensis ATCC 27448: Modulation of pimaricin production by intracellular ROS. PLOS ONE 2011, 6, e27472. [CrossRef]

41. Miranda, R.U.; Gómez-Quiroz, L.E.; Mendoza, M.; Pérez-Sánchez, A.; Fierro, F.; Barrios-González, J. Reactive oxygen species regulate lovastatin biosynthesis in Aspergillus terreus during submerged and solid-state fermentations. Fungal Biol. 2014, 118, 979-989. [CrossRef]

42. Bibián, M.E.; Pérez-Sánchez, A.; Mejía, A.; Barrios-González, J. Penicillin and cephalosporin biosyntheses are also regulated by reactive oxygen species. Appl. Microbiol. Biotechnol. 2020, 104, 1773-1783. [CrossRef] [PubMed]

43. Prajapati, D.; Kumari, N.; Dave, K.; Chatupale, V.; Pohnerkar, J. Chromomycin, an antibiotic produced by Streptomyces flaviscleroticus might play a role in the resistance to oxidative stress and is essential for viability in stationary phase. Environ. Microbiol. 2019, 21, 814-826. [CrossRef] [PubMed]

44. Thompson, C.J.; Ward, J.M.; Hopwood, D.A. DNA cloning in Streptomyces: Resistance genes from antibiotic-producing species. Nature 1980, 286, 525-527. [CrossRef] [PubMed]

45. Young, M.; Artsatbanov, V.; Beller, H.R.; Chandra, G.; Chater, K.F.; Dover, L.G.; Goh, E.-B.; Kahan, T.; Kaprelyants, A.S.; Kyrpides, N.; et al. Genome sequence of the Fleming strain of Micrococcus luteus, a simple free-living actinobacterium. J. Bacteriol. 2010, 192, 841-860. [CrossRef]

46. Szczerba, I. Susceptibility to antibiotics of bacteria from genera Micrococcus, Kocuria, Nesterenkonia, Kytococcus and Dermacoccus. Medycyna Doświadczalna i Mikrobiologia 2003, 55, 75-80.

47. R.Core Team. A Language and Environment for Statistical Computing; R Foundation for Statistical Computing: Vienna, Austria, 2013.

48. Hervé, M. RVAideMemoire: Testing and Plotting Procedures for Biostatistics. R Package Version 0.9-75. 2020. Available online: https://CRAN.R-project.org/package=RVAideMemoire (accessed on 4 March 2020).

49. Folch, J.; Lees, M.; Sloane Stanley, G.H. A simple method for the isolation and purification of total lipides from animal tissues. J. Biol. Chem. 1957, 226, 497-509.

50. Abreu, S.; Solgadi, A.; Chaminade, P. Optimization of normal phase chromatographic conditions for lipid analysis and comparison of associated detection techniques. J. Chromatogr. A 2017, 1514, 54-71. [CrossRef]

51. Dixon, R.W.; Peterson, D.S. Development and testing of a detection method for liquid chromatography based on aerosol charging. Anal. Chem. 2002, 74, 2930-2937. [CrossRef]

52. Graves, S.; Piepho, H.-P.; Selzer, L.; Dorai-Raj, S. multcompView: Visualizations of Paired Comparisons. R Package Version 0.1-8. 2019. Available online: https:/CRAN.Rproject.org/package=multcompView (accessed on 19 December 2019).

53. Wick, R.R.; Judd, L.M.; Gorrie, C.L.; Holt, K.E. Unicycler: Resolving bacterial genome assemblies from short and long sequencing reads. PLoS Comput. Biol. 2017, 13, e1005595. [CrossRef]

54. Moriya, Y.; Itoh, M.; Okuda, S.; Yoshizawa, A.C.; Kanehisa, M. KAAS: An automatic genome annotation and pathway reconstruction server. Nucleic Acids Res. 2007, 35, W182-W185. [CrossRef]

55. Altschul, S.F.; Gish, W.; Miller, W.; Myers, E.W.; Lipman, D.J. Basic local alignment search tool. J. Mol. Biol. 1990, 215, 403-410. [CrossRef]

(C) 2020 by the authors. Licensee MDPI, Basel, Switzerland. This article is an open access article distributed under the terms and conditions of the Creative Commons Attribution (CC BY) license (http://creativecommons.org/licenses/by/4.0/). 